\title{
Description of muzzle blast by modified ideal scaling models
}

\author{
Kevin S. Fansler \\ U.S. Army Research Laboratory, Aberdeen Proving \\ Ground, MD 21005, USA
}

Gun blast data from a large variety of weapons are scaled and presented for both the instantaneous energy release and the constant energy deposition rate models. For both ideal explosion models, similar amounts of data scatter occur for the peak overpressure but the instantaneous energy release model correlated the impulse data significantly better, particularly for the region in front of the gun. Two parameters that characterize gun blast are used in conjunction with the ideal scaling models to improve the data correlation. The gun-emptying parameter works particularly well with the instantaneous energy release model to improve data correlation. In particular, the impulse, especially in the forward direction of the gun, is correlated significantly better using the instantaneous energy release model coupled with the use of the gun-emptying parameter. The use of the Mach disc location parameter improves the correlation only marginally. A predictive model is obtained from the modified instantaneous energy release correlation.

\section{Introduction}

Before guns are fired near personnel or fragile equipment that might be harmed, blast wave overpressure levels need to be accurately known over a large range of distances from the gun muzzle. Personnel and instruments may need to be located as close as 15 to 20 calibers away from a gun. On the other hand, designing an enclosure for reducing impulsive noise may require an estimate of the forces and impulses on its inside surfaces as far away as 100 calibers from the muzzle. Some of the quantities that can be used to assess gun blast at a field point are the peak overpressure, $p_{\mathrm{p}}$, the time of arrival for the front of the blast wave, $t_{\mathrm{a}}$, the impulse, $I$, and the positive phase duration, $\tau$. The positive phase duration is the time for the positive phase of the wave to pass over the field point. The time of arrival and positive phase du- ration need to be known accurately when peak overpressures and impulses at a field point result from multiple reflections of the blast wave.

The impulse, $I$, is defined as the time integral of the positive phase for the overpressure:

$$
I \equiv \int_{t_{\mathrm{a}}}^{t_{\mathrm{c}}}\left(p-p_{\infty}\right) \mathrm{d} t
$$

in which $t_{\mathrm{c}}$ is the time for the overpressure to become zero in the blastwave. Here $p$ is the pressure and $p_{\infty}$ is the atmospheric pressure, which near sea level is approximately one bar. The impulse is a very important quantity in determining if structures are reinforced adequately to withstand the blast wave.

The first predictions of gun blast were done using results obtained from instantaneous energy release blast from a point source. The Buckingham Pi theorem can be applied to instantaneous energy release at a point source to obtain a fundamental length, $\lambda$ (Baker [2]). The scaled peak overpressure, $\bar{P}$, becomes a function of the distance scaled by the fundamental length (Hopkinson [11]; Baker [2]). Although explosive charges will not strictly generate point source blasts, they approximate blast waves from these ideal point source blasts after a minimum scaled distance is reached (Brode [3]). Reynolds [14] applied this point source scaling theory developed by Hopkinson [11] to gun blast problems. Westine [18] proposed a scaling prediction method based on highexplosive detonations in combination with the length of the gun barrel. He based his work primarily on $20 \mathrm{~mm}$ data that included different projectile muzzle velocities and developed contours for predicting peak overpressure and time of arrival for a wide range of weapons, but these contours did not extend completely around the weapons.

Smith [16] used a different approach in his study of the blast wave produced by a $7.62 \mathrm{~mm}$ rifle. He combined scaled solutions to the problems of blast waves generated by a constant energy efflux with blast waves from asymmetrically initiated charges. The theory of asymmetrically initiated charges was applied 
to account for the peak overpressure values decreasing with increasing polar angle, with zero polar angle being along the boreline of the gun. The prediction method agreed well with the limited data.

Schmidt, Gion, and Fansler [15] used yet another approach to predicting muzzle blast. They found that the peak overpressure correlated well with the location of the Mach disc for an equivalent steady jet whose exit conditions matched that for the pressure and the propellant gas velocity. Later, Fansler and Schmidt [8] found that the Mach disc method of prediction was deficient in its ability to predict the peak overpressure changes with the change in the propellant temperature. However, Smith's [16] prediction model predicted the actual trends. Fansler and Schmidt [8] generalized and extended Smith's work [16] to develop prediction methods for bare muzzle guns, based on data collected in the range of 10 to 50 calibers from the gun muzzle. From dimensional analysis, a scaling length, $\xi$, was obtained that is assumed to be proportional to the square root of the peak energy efflux from the gun muzzle. This scaling length depends on such parameters as the exit muzzle pressure, exit temperature of the propellant, and the projectile velocity at the gun muzzle. The distance from the muzzle to the field point divided by the scale length, $r / \xi$, yields the fundamental scaled distance. However, the blast waves are highly directional, with their peak overpressures decreasing with increasing polar angle from the forward axis direction. The form for the variation of peak overpressure with angle from the axis is obtained from asymmetrically initiated charges, or equivalently, moving charge theory (Armendt and Sperrazza [1]). This angle variation function, $\beta$, possesses one free parameter that determines how rapidly the peak overpressure falls off with increasing polar angle. The function, $\beta(\theta)$, is multiplied by the fundamental scale length, $\xi$, to obtain the modified scaled length, $\xi^{\prime}$, for gun blast. The assumed formulation for the scaled peak overpressure was

$$
\bar{P}=A\left(\frac{\xi^{\prime}}{r}\right)^{a},
$$

in which $r$ is the distance from the muzzle and the free parameters, $A$ and $a$ together with the free parameter for $\beta$, are determined by a least squares fit to peak overpressure data. The other blast wave quantities of interest were also formulated and least square fitted to data.

The predicted muzzle blast quantities were implemented on a computer and can be applied for blast waves incident on surfaces to obtain the reflected pressures (Heaps et al. [10]). Fansler [6], noting deficiencies in the impulse model, examined additional impulse data and developed an expression that depended not only on the scaling length but also on a dimensionless parameter that depended upon the time for the gun barrel to empty. The computer-implemented technique (Heaps et al. [10]) was updated with the improvement for treating impulse.

In the studies just cited, data were collected for distances close to the muzzle. Other people have investigated muzzle blast at greater distances. Soo Hoo and Moore [17] primarily studied various naval guns with data taken for distances between 20 and 110 calibers but also obtained data for U.S. Army $20 \mathrm{~mm} \mathrm{M3}$ and M197 cannon. Kietzman, Fansler, and Thompson [12] obtained overpressure data for a maximum of 400 calibers distance from a $105 \mathrm{~mm}$ tank cannon and noted that the angular distribution of the shock wave strength changed with distance. Pater [13] obtained additional data and combined his data with Soo Hoo and Moore's data. He also noted that the peak sound pressure level (PSPS) decibel differences from the front of the gun to the rear of the gun decreased with distance.

Fansler et al. [9] obtained data from $7.62 \mathrm{~mm}$ rifles for a large range of distances (15 to 400 calibers) from the muzzle and for weapons shot at both high and low velocities with different lengths of barrels. The approach was similar to Fansler and Schmidt's [8] approach but explored the use of gun blast parameters to modify the directional parameter, $\beta$. The data were fitted using several trial parameters and functions to obtain a best representative function for the peak overpressure. Time-of-arrival data were also obtained and a fit was obtained from a trial function. The impulse prediction function was obtained by assuming a positive phase duration form and expressing the impulse in terms of the peak overpressure and the positive phase duration. The positive phase duration possessed free parameters to be adjusted by a least squares fit to the impulse data.

Previous investigators usually developed a model assuming only one approach. This report reconsiders both the point source scaling approach and the energy efflux scaling approach as a basis for modification by the parameters that characterize gun blast. The length scale for the chosen ideal explosion is modified by parameters for the gun blast, and coefficients for these parameters are determined by least squares fitting to the data. This study assumes that functions of the gun blast parameters will be good first order corrections to the scale length for the ideal models. 


\section{Data used in investigation}

Most previous scaling investigations conducted used data with less variation in key parameters (Fansler and Schmidt [8]; Smith [16]; Reynolds [14]) than are used in the current investigation. Thus, an earlier prediction model for gun blast might fit the earlier (limited) data but it might be a poor fit for other data used here. This investigation uses much of the Fansler et al. [9] data, but in an attempt to more accurately model the data nearer the gun, ignores the data taken at the longest distance, which was 400 calibers away from the gun muzzle. This investigation also uses some of the data of Fansler and Schmidt [8] and Kietzman, Fansler, and Thompson [12] to provide a greater range in gun blast parameters. In all these experiments, a gauge was positioned near the muzzle to establish a zero reference time for the experiment. The experiment to obtain the small-caliber overpressure data $(7.62 \mathrm{~mm})$ was conducted at ARL for distances 15 to 400 calibers from the muzzle (Fansler et al. [9]). For the current study, the data at 400 calibers will not be used. The weapons used in the test were a .300 Magnum barrel, another .300 Magnum barrel that had a reduced bore length, and a shortened carbine barrel. A schematic of the gauge positions around the gun muzzles is shown in Fig. 1 for the investigation by Fansler et al. [9].

In addition, $105 \mathrm{~mm}$ tank cannon data obtained in the Kietzman, Fansler, and Thompson [12] investigation are included. Some $30 \mathrm{~mm}$ WECOM data from Fansler and Schmidt [8] are also included because they can be used to extend the range of applicability to more gun systems. The propellant for the $30 \mathrm{~mm}$ WECOM cannon was specially selected to burn quickly in the barrel to maximize reproducibility. The propellant speed of sound at the muzzle was also experimentally found.

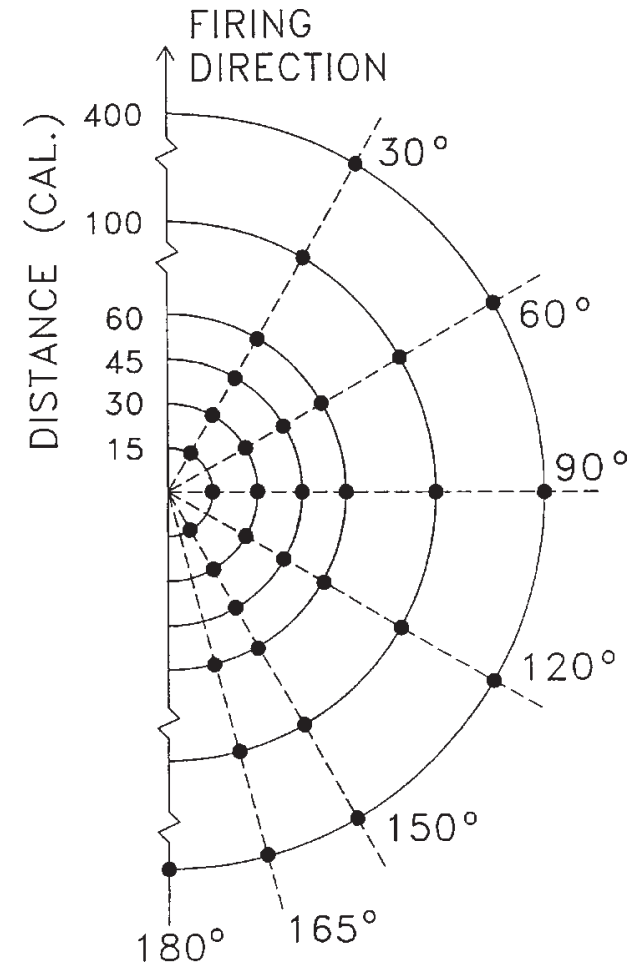

Fig. 1. Gauge positions for the Fansler et al. study [9].

The loading, velocity of the projectile at the muzzle, and muzzle pressure at projectile exit are shown in Table 1 for each configuration. The muzzle pressure in the last column is the peak value immediately before the projectile exits the barrel. With the exception of the last two rows, the first number in the description column of Table 1 refers to the charge mass in grains, while the second number refers to the charge mass in grams. Further discussions about the firings will use these descriptions for identification. The next to the last row refers to the parameters for the $105 \mathrm{~mm}$ cannon shooting the M735 round (Kietzman et al. [12]).

Table 1

Loadings and characteristics

\begin{tabular}{|c|c|c|c|c|c|c|}
\hline \multirow[t]{2}{*}{ Barrel } & \multirow[t]{2}{*}{ Description } & \multirow[t]{2}{*}{$\begin{array}{c}\text { Propellant } \\
\text { type }\end{array}$} & \multicolumn{2}{|c|}{$\begin{array}{c}\text { Charge } \\
\text { mass }\end{array}$} & \multirow{2}{*}{$\begin{array}{c}\text { Projectile } \\
\text { velocity } \\
\mathrm{m} / \mathrm{s}\end{array}$} & \multirow{2}{*}{$\begin{array}{c}\text { Muzzle } \\
\text { pressure } \\
\mathrm{MPa}\end{array}$} \\
\hline & & & $\mathrm{g}$ & (gr) & & \\
\hline \multirow[t]{2}{*}{.300 Long Magnum } & $1220 w 70$ & 4831 & 4.82 & $(70.0)$ & 899 & 59.40 \\
\hline & $1125 w 75$ & 4831 & 5.16 & $(75.0)$ & 975 & 71.90 \\
\hline \multirow{2}{*}{.300 Short Magnum } & s220w36 & 4227 & 2.48 & $(36.0)$ & 594 & 74.90 \\
\hline & s $125 w 42$ & 4227 & 2.89 & $(42.0)$ & 792 & 89.60 \\
\hline Carbine & c13p6 & 2400 & 0.94 & $(13.6)$ & 518 & 65.90 \\
\hline $105 \mathrm{~mm}$ & $105 \mathrm{~mm}$ & M30 & 5966 & $(-)$ & 1501 & 71.50 \\
\hline $30 \mathrm{~mm}$ & $\mathrm{~m} 10 \mathrm{f} 250$ & M10 & 16.2 & $(250)$ & 572 & 8.73 \\
\hline
\end{tabular}




\section{Scaling approaches}

Because the propellant empties from the barrel in a time that depends on parameters such as gun barrel length, and the propellant exits the barrel with considerable linear momentum, no simple ideal scaling theory (e.g., neither instantaneous nor constant energy release models) can be successfully applied to gun blast phenomena without some modifications. Nevertheless, even though the blast wave from guns is more complicated, the similarities to a particular ideal explosion may allow a related scaling technique to be used with modification by gun blast parameters to represent gun blast waves. Both instantaneous energy release explosions and constant energy efflux explosions are treatable by scaling and have been used to help describe gun blast (Reynolds [14]; Westine [18]; Fansler and Schmidt [8]; Fansler et al. [9]). For blasts generated by a constant energy efflux, $\mathrm{d} E / \mathrm{d} t$, the peak overpressure, $P \equiv p_{\mathrm{p}}-p_{\infty}$, is expressed in functional terms as

$$
P=P\left(r, \rho_{\infty}, a_{\infty}, \mathrm{d} E / \mathrm{d} t\right),
$$

in which $p_{\mathrm{p}}=$ peak value of the pressure at a given field point, $r=$ distance from muzzle to field point, $\rho_{\infty}=$ ambient density, $a_{\infty}=$ ambient speed of sound, and $\mathrm{d} E / \mathrm{d} t=$ energy deposition rate into atmosphere.

Using the Buckingham Pi theorem (Baker [2]), a scaling length for constant energy efflux explosion is obtained:

$$
\xi \sim \sqrt{(\mathrm{d} E / \mathrm{d} t) /\left(p_{\infty} a_{\infty}\right)}
$$

and

$$
\bar{P} \equiv P / p_{\infty}=\bar{P}(r / \xi) .
$$

Dimensional analysis shows that the time of arrival, $t_{\mathrm{a}}$, the positive phase duration, $\tau$, and the impulsive, $I$, also have their scaled equivalents,

$$
\begin{gathered}
\bar{t}_{\mathrm{a}} \equiv t_{\mathrm{a}} a_{\infty} / \xi=\bar{t}_{\mathrm{a}}(r / \xi), \\
\bar{\tau} \equiv \tau a_{\infty} / \xi=\bar{\tau}(r / \xi),
\end{gathered}
$$

and

$$
\bar{I} \equiv I a_{\infty} /\left(\xi p_{\infty}\right)=\bar{I}(r / \xi) .
$$

For blast waves assumed to be generated by instantaneous energy deposition at a source point, dimensional analysis leads to scaling relationships that also generate universal curves, but with the energy of the explosive, $E$, considered a significant parameter in- stead of $\mathrm{d} E / \mathrm{d} t$. Similarly, as for the constant energy efflux case, it is obtained that

$$
\bar{P}=\bar{P}(r / \lambda),
$$

in which

$$
\lambda=\left(\frac{E}{p_{\infty}}\right)^{1 / 3} .
$$

The point source expressions for the scaled time, positive phase duration, and impulse are Eqs (6), (7), and (8) with $\xi$ replaced by $\lambda$.

As related before, gun blast cannot be completely characterized by either of these ideal blast models. Nevertheless, the ideal models can be modified to describe muzzle blast. The energy release rate of propellant gas from the gun muzzle exit depends upon the length of the barrel, the velocity of the projectile, and the sound speed of the propellant gas before projectile exit. As a first step in characterizing muzzle blast in terms of the constant energy efflux model, the energy deposition rate for gun blast is assumed to be the energy efflux from the muzzle exit immediately after the projectile back clears the muzzle exit. The energy deposition rate can then be written as

$$
\frac{\mathrm{d} E}{\mathrm{~d} t}=\frac{\gamma_{\mathrm{e}} p_{\mathrm{e}} u_{\mathrm{e}}}{\gamma_{\mathrm{e}}-1}\left[1+\frac{\left(\gamma_{\mathrm{e}}-1\right)}{2} M_{\mathrm{e}}^{2}\right] A_{\mathrm{e}},
$$

in which $A_{\mathrm{e}}$ is the area of the bore, $M_{\mathrm{e}}$ is the exit Mach number of the propellant flow immediately after the projectile exits the muzzle, $p_{\mathrm{e}}$ is the peak muzzle overpressure while the projectile exits the muzzle, $u_{\mathrm{e}}$ is the velocity of the propellant gas at the exit immediately after the projectile exits the muzzle, and $\gamma_{\mathrm{e}}$ is the specific heat ratio for the exiting propellant.

To use the instantaneous energy release model as a basis for gun blast, a value for $E$ must be assumed. The available energy, $E$, is assumed to be the total energy in the propellant minus the energy expended in propelling the projectile and heating the gun tube by friction and heat transfer. The kinetic energy of the propellant gas is part of the available energy for the blast.

In addition to prescribing $\mathrm{d} E / \mathrm{d} t$ and $E$ (for use in the constant energy efflux and point source models, respectively), other dimensionless parameters are needed to modify the ideal models for accurately computing gun blast. For instance, if a gun barrel empties quickly, the peak overpressure will be higher than if the energy is released over a longer period of time. Neither the initial $\mathrm{d} E / \mathrm{d} t$ nor $E$ itself will account for this emptying time effect. Hence, an additional pa- 
Table 2

Blast parameters for the point source and energy efflux models

\begin{tabular}{cccrcr}
\hline Designation & $\lambda / D$ & $\delta_{\lambda}$ & $\xi / D$ & $\delta_{\xi}$ & $x_{\mathrm{M}} / D$ \\
\hline c13p6 & 39.8 & 0.437 & 9.44 & 1.84 & 15.80 \\
s220w36 & 51.4 & 0.712 & 9.45 & 3.87 & 17.60 \\
s125w42 & 55.6 & 0.462 & 10.40 & 2.47 & 22.30 \\
1220w70 & 60.1 & 0.694 & 8.41 & 4.96 & 25.60 \\
1125w75 & 63.8 & 0.598 & 9.80 & 3.89 & 26.60 \\
105mm & 54.9 & 0.214 & 14.01 & 0.82 & 38.90 \\
m10f250 & 22.2 & 0.955 & 3.08 & 6.88 & 6.58 \\
\hline
\end{tabular}

rameter characterizing the blowdown process, which can be obtained with the Buckingham Pi theorem, is used (Fansler [6]; Baker [2]).

For the constant energy release model, the blowdown parameter is defined by the ratio of the scale for the gun tube emptying time, $L / V_{\mathrm{p}}$ (Corner [4]), to the time scale for muzzle blast, $\xi / a_{\infty}$.

$$
\delta_{\xi} \equiv \frac{L a_{\infty}}{\xi V_{\mathrm{p}}},
$$

in which $L$ is the effective length of the barrel and $V_{\mathrm{p}}$ is the exit velocity of the projectile. The smaller the value of $\delta_{\xi}$, the more quickly the barrel is emptied. Its counterpart for the instantaneous energy release model is

$$
\delta_{\lambda} \equiv \frac{L a_{\infty}}{\lambda V_{\mathrm{p}}}
$$

Again, when the blow-down parameter is small, the barrel empties more quickly, which would result in the energy from the propellant being used in a more efficient manner by the gun blast wave. These expressions for the blow-down parameter are not unique but they are simple and may be adequate representations for times to empty guns.

Another parameter that characterizes gun blast is the axial location of the Mach disc or the recompression shock that is centered on the gun bore axis (Erdos and Del Guidace [5]; Schmidt et al. [15]). For the steady jet, the axial position of the Mach disc relative to the muzzle for the steady jet is given as

$$
x_{\mathrm{M}}^{\prime} / D=M_{\mathrm{e}} \sqrt{\gamma_{\mathrm{e}} \bar{p}_{\mathrm{e}} / 2},
$$

in which $D$ is the diameter of the bore. The Mach disc location expression resembles the constant energy efflux expression but differs in the emphasis upon the Mach number for the exit flow and the exit velocity for the muzzle flow. The use of the Mach disc location as a gun parameter allows other characteristics of the gun blast flow to be expressed. It has been used with some success as a scaling factor (Schmidt et al. [15]). The scale lengths for the ideal types of explosions, the blow-down parameters, and the Mach disc locations are given in Table 2 for various gun test data presented in this paper.

\section{Results}

A computer program was developed to extract the peak overpressure, the impulse (numerically calculated time integral of the positive phase for the overpressure), and the time of arrival from the obtained overpressure waveforms. Then, both the scaled peak overpressure data and the scaled impulse data is scaled with the fundamental length for each model and presented for the polar angles of $30^{\circ}, 90^{\circ}$, and $150^{\circ}$. If the two models are comparable in their correlations, then modification of the scale length with the gun blast parameters are attempted by least square fitting to obtain improved correlations. Otherwise, if a correlation is superior for one of the ideal scaling models, then only that model will be selected to improve correlation of the data with the use of the gun blast parameters.

\subsection{Peak overpressure data presented with different scaling approaches}

The data can first be examined along selected rays when the distance from the gun tube muzzle is scaled by $\xi$, corresponding to an explosion generated by a constant energy efflux. Figure 2 shows the peak over-

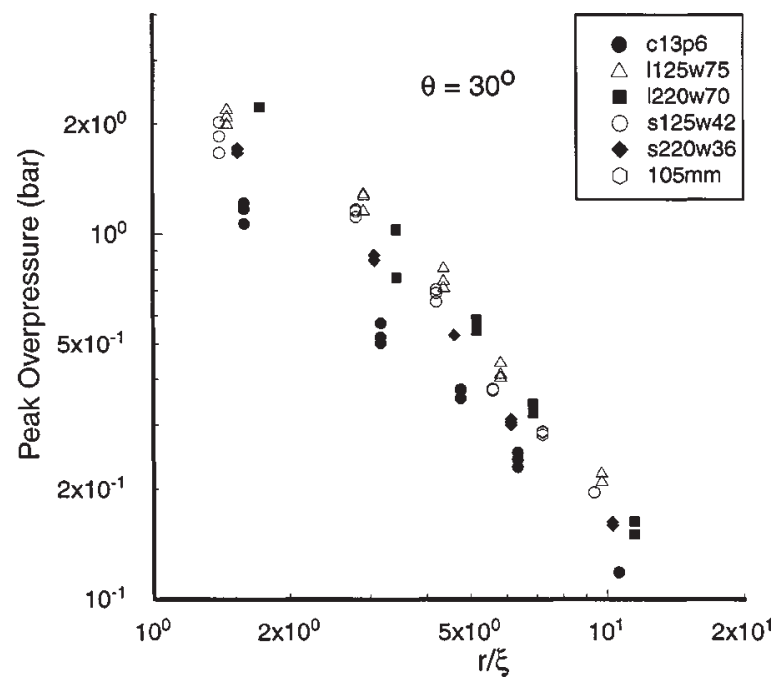

Fig. 2. Scaled peak overpressure data for $\theta=30^{\circ}$ (energy efflux assumption). 


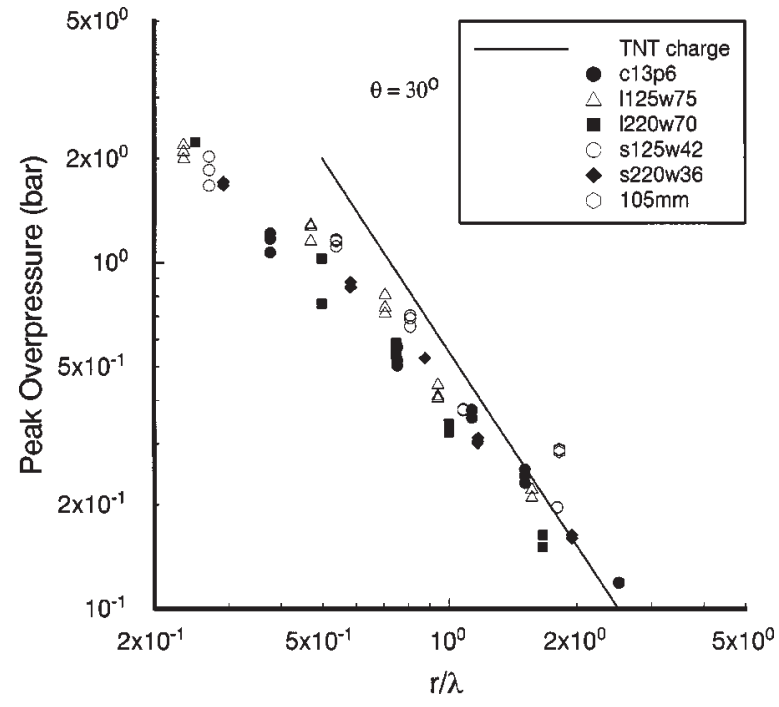

Fig. 3. Scaled peak overpressure data for $\theta=30^{\circ}$ (point source assumption) and calculation for TNT (Brode [3]).

pressure data along the ray directed $30^{\circ}$ from the muzzle axis.

When the data are scaled with the peak energy efflux approach, the slower emptying weapons (more resembling constant energy efflux) should have higher values of peak overpressure relative to the faster emptying weapons (less resembling constant energy efflux). Least square fitting, discussed later in this section, will show that the weapons with the largel muzzle to Mach disc distances also tend to generate larger peak overpressures, given equal values of $\delta_{\gamma}$. The Mach disc location parameter is used because it was found to correlate data (Schmidt et al. [15]), but a physical reason why this parameter could be used to improve correlation for either the constant energy efflux model or the point blast model is not known.

The results for Fig. 2 indicate that the Mach disc position will correlate the data better than the blowdown parameter, when scaled for the constant energy efflux model. Nevertheless, the rapid blowdown for the $105 \mathrm{~mm}$ cannon appears to bring the data points into close company with other larger data values, even though the muzzle-to-Mach disc distance is larger than for any other firings. The c13p6 data have values for their parameters that would predict that the peak overpressure would be low compared to all the other data. Similar behavior occurs for data taken at other angles and are discussed in detail by Fansler [7].

The peak overpressure data are next examined with the distance scaled by $\lambda$, corresponding to the point source explosion. Figure 3 shows the peak overpres- sure data along the $30^{\circ}$ ray. Also shown is a calculation for a spherical TNT charge in open air. The TNT calculations were obtained with a finite difference scheme developed by Brode [3]. The calculated values for the TNT charge should better approximate the ideal point source solution as the distance increases from the TNT charge. It is seen that the peak overpressure for the TNT charge is initially higher but decreases so rapidly that it is less than some of the gun blast data values at the longer scaled distances. Recall that smaller values of $\delta_{\lambda}$ are associated with a quicker emptying time (more resembling a point source) and should have higher peak overpressure values for a given distance, which generally occurs. Some exceptions result from data scatter, the other reasons are not known. The trends are generally similar for data at other polar angles (Fansler [7]). Both unmodified approaches show deficiencies in correlating the data. It is not clear that either approach, when modified by the characteristic gun parameters, would be noticeably superior to the other. Accordingly, least squares fitting are performed for both the point source model and the energy efflux model.

\subsection{Modeling approach for peak overpressure data}

The ideal scaling relationships, which assume spherically symmetrical conditions, need to be modified for gun blast, where the peak overpressure varies strongly with the polar angle, $\theta$, from the bore-line. Following Smith [16], a directional scaling length factor is obtained, $\beta$, that depends on the polar angle,

$$
\beta=\mu \cos \theta+\sqrt{1-\mu^{2} \sin ^{2} \theta},
$$

in which $\mu$ is the momentum index, which determines the levels of peak overpressure (with distance held constant) as a function of the polar angle, $\theta$. This expression has been developed from moving blast theory. The momentum of the charge results in the strength of the blast being increased in the direction of the momentum and decreased to the rear of the moving charge. The directional length scaling factor, $\beta$, is real and positive if $\mu<1$. The nearer $\mu$ is to zero, the more nearly spherical the blast. As $\mu$ approaches 1, the strength of the blast in front of the gun becomes large compared to the strength of the blast wave to the rear.

To improve the prediction model, the scaling lengths, $\xi$ and $\lambda$, need to be modified with the two gun blast parameters discussed earlier. Trial functions of these parameters are assumed as first order terms 
in a series expansion. Least square fitting showed that the most effective functions of these parameters involved the inverse of the blow-down parameter and the first power of the Mach disc location. Along with $\beta$ to be determined by least squares fitting in terms of $\mu$, two other constants that multiply these parameter functions need to be fitted to obtain the modified scaling lengths, $\xi^{\prime}$ and $\lambda^{\prime}$, defined by

$$
\begin{aligned}
& \lambda^{\prime} \equiv \lambda \beta\left(1+\frac{C}{\delta_{\lambda}}+H \frac{x_{\mathrm{M}}}{D}\right), \\
& \xi^{\prime} \equiv \xi \beta\left(1+\frac{C}{\delta_{\xi}}+H \frac{x_{\mathrm{M}}}{D}\right) .
\end{aligned}
$$

With these modifications, the gun blast peak overpressure is

$$
\bar{P}=\bar{P}(\bar{r})
$$

in which $\bar{r} \equiv r / \ell$, and $\ell$ assumes the value of $\xi^{\prime}$ or $\lambda^{\prime}$ depending on the scaling approach used.

Also, the time of arrival, $t_{\mathrm{a}}$, has its scaled equivalent,

$$
\bar{t}_{\mathrm{a}}(r / \ell) \equiv t_{\mathrm{a}} a_{\infty} / \ell .
$$

The wave generated from gun blast decays to an acoustic wave $(\bar{P} \propto 1 / r)$ at large distances, but closer in, the peak overpressure versus distance relationship has a steeper slope $\left(\bar{P} \propto 1 / r^{a}, a>1\right)$, as also occurs with instantaneous explosions. It is attempted to model this behavior with the following expression for the peak overpressure, $\bar{P}$,

$$
\bar{P}=\frac{A}{\bar{r}}+\frac{B}{\bar{r}^{2}} .
$$

Another more simple model will also be tried and compared with the two-term model:

$$
\bar{P}=\frac{A}{\bar{r}^{a}},
$$

in which $a$ is assumed to be constant throughout the region of interest. Equation (20) or Eq. (21) is matched with pressure data in conjunction with Eq. (15), which is the expression used to vary the strength of the blast with the polar angle, $\theta$. The values of the significant parameters, $\lambda / D, \delta_{\lambda}, \xi / D, \delta_{\xi}$, and $X_{\mathrm{M}} / D$, are given in Table 2. With these values and the peak overpressure data, fits are made to determine $A, B, a$, and $\mu$ for the one-term model, $C$, and $H$.

\subsection{Peak overpressure fitting}

Fits were made for both the point source model and the energy efflux model with the use of the blowdown parameters and Mach disc position. For the point source model, Table 3 gives the parameter values found for the predictive equations for both the one-term equation and the two-term equations.

The particular headings refer to the symbols occuring in Eqs (15), (16), (17), (20), and (21). The oneterm models (1pa, $1 \mathrm{pb}, 1 \mathrm{pc}$, and $1 \mathrm{pd})$ are presented first in Table 3 , in which $B=0$ and $a \neq 1$. When $C$ and $H$ are assumed to be zero in the fitting procedure, the root mean square (RMS) error is 0.309 . This value is obtained from fitting the logarithm of the pressure data with the logarithm of the fitting function. The RMS value of 0.309 translates to an expectation that the data value will be $36 \%$ more or less than the curve fit value. Fitting with the inverse blow-down parameter (model 1pc) gives good improvement while fitting with Mach disc position (model $1 \mathrm{pb}$ ) yields significantly less improvement. Concurrent fitting with the blow-down and Mach disc position parameters gives only marginal improvement over fitting with the blowdown parameter. The two-term models (2pa and $2 \mathrm{pb}$ ) give comparable results. The physical intuition that the scaled (point mass scaling) peak overpressures with longer blow-down times would be smaller relative to the peak overpressures for smaller blow-down times is confirmed by the positive fitted values for $C$.

The results for the energy efflux model are presented in Table 4. The important parameter to im-

Table 3

Least squares fit results for peak overpressure data (point source model)

\begin{tabular}{cccccccc}
\hline Model & $\mu$ & $A$ & $B$ & $C$ & $H$ & $a$ & RMS \\
\hline $1 \mathrm{pa}$ & 0.78 & 0.270 & - & - & - & 1.10 & 0.309 \\
$1 \mathrm{pb}$ & 0.78 & 0.145 & - & - & 0.0374 & 1.09 & 0.247 \\
$1 \mathrm{pc}$ & 0.76 & 0.117 & - & 0.562 & - & 1.14 & 0.199 \\
$1 \mathrm{pd}$ & 0.77 & 0.100 & - & 0.564 & 0.0159 & 1.13 & 0.193 \\
$2 \mathrm{pa}$ & 0.78 & 0.243 & 0.0220 & - & - & - & 0.309 \\
$2 \mathrm{pb}$ & 0.78 & 0.110 & 0.0061 & 0.592 & - & - & 0.201 \\
\hline
\end{tabular}

Table 4

Least squares fit results for peak overpressure data (energy efflux model)

\begin{tabular}{cccccccc}
\hline Model & $\mu$ & $A$ & $B$ & $C$ & $H$ & $a$ & RMS \\
\hline 1ea & 0.77 & 1.89 & - & - & - & 1.13 & 0.252 \\
1eb & 0.78 & 1.14 & - & - & 0.0248 & 1.10 & 0.208 \\
1ec & 0.77 & 1.77 & - & 0.107 & - & 1.12 & 0.251 \\
1ed & 0.77 & 1.18 & - & -0.349 & 0.0299 & 1.12 & 0.201 \\
2ea & 0.78 & 1.31 & 0.742 & - & - & - & 0.252 \\
2eb & 0.78 & 0.89 & 0.247 & - & 0.0271 & - & 0.210 \\
\hline
\end{tabular}


prove fitting for the energy efflux model is the Mach disc position instead of the inverse blow-down parameter, $1 / \delta_{\xi}$, as it was for the point source model. The fit marginally improved with the use of the inverse blow-down parameter. The fit performed to fit values for both $C$ and $H$ (model led) gives a negative value for $C$ and confirms the intuition that longer blowdown times result in higher scaled peak overpressures with the constant energy efflux model. The positive value obtained for $C$ when $H$ is assumed to be zero (1ec) occurs because there is a correlation between the blowdown and the Mach disc location parameter values. For a given gun configuration, increasing the charge mass results in a larger initial energy efflux and a smaller $\delta_{\xi}$. The trend is noted in Table 2. When $H$ is assumed to be zero (model 1ec), the effect of the Mach disc location overshadows the blow-down parameter and produces a false illusion. For both the point source model and the energy efflux model, other fits were attempted with the gun parameters raised to other powers in Eqs (16) and (17). These results are not shown as these particular choices yielded noticeably larger root mean square errors than those shown.

The use of the blow-down parameter with the point source model significantly improves the data correlation while the use of the Mach disc position parameter with the constant energy efflux model markedly improves the data correlation. The point source model was selected as the basis for modification because, as will be seen in a later section, point source scaling correlates the impulse data better than the energy efflux scaling does. In terms of correlation, there is little to choose between the two-term modified point source model and the one-term modified point source model. The two-term modified point source model was selected in preference to the one-term model because the ratio of the peak overpressure to the front of the gun over the peak overpressure to the rear of the gun becomes larger nearer the gun muzzle, as experiment shows. Also, the expression for the time of arrival is simplified by the use of the two-term point source model with the blow-down parameter modification as compared with the use of the one-term expression. The selected fit to calculate future predicted fits for the peak overpressure is model $2 \mathrm{pb}$ in Table 3 , which gives

$$
\bar{P}=0.11 \frac{\lambda^{\prime}}{r}+0.0061\left(\frac{\lambda^{\prime}}{r}\right)^{2},
$$

is which $\lambda^{\prime}$ is given by Eq. (16), which varies with $\theta$ through $\beta$, as given by Eq. (15). The scaled data with

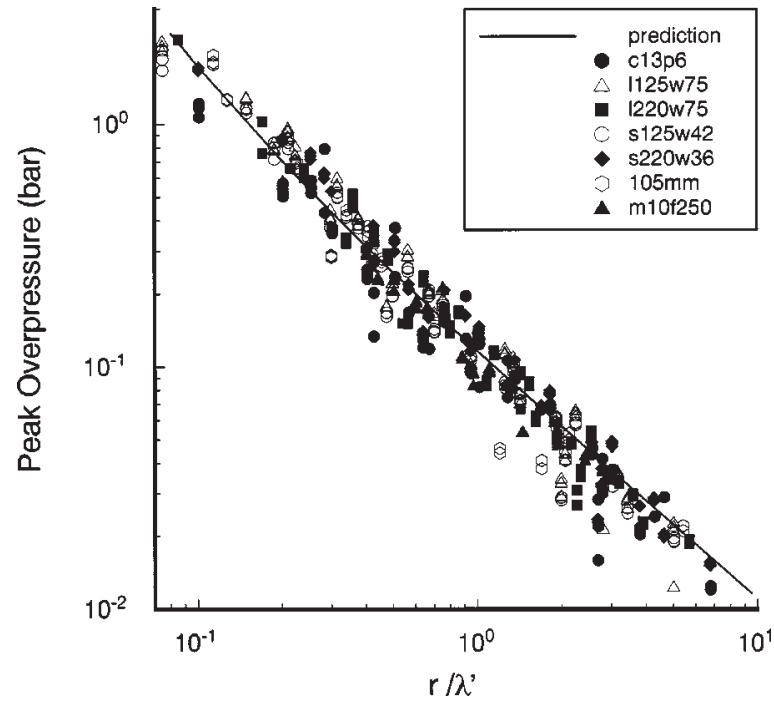

Fig. 4. Peak overpressure versus $r / \lambda^{\prime}$ with least squares fit.

the prediction curve is shown in Fig. 4. As expected, the carbine data (c13p6) show the most scatter, which may have to do with their gun blast parameter values. The parameters for the carbine are an unusual combination when compared with the other weapons. The carbine has the second smallest $\delta_{\lambda}$ and the second from the smallest muzzle-to-Mach disc distance in the group. If the other weapon's value of $\delta_{\lambda}$ were placed in increasing order, the value of $x_{\mathrm{M}} / D$ usually appears in declining order. Future studies should include weapons with similar characteristics to the carbine (c13p6).

Equation (22) gives good agreement over the parameter ranges where data were obtained. One should employ caution in extrapolating to parameter values outside the range where data were taken.

\subsection{Modeling and fitting time-of-arrival data}

As in Fansler and Schmidt [8], the pressure-jump Mach relation,

$$
\bar{P}=\frac{2 \gamma}{\gamma+1}\left(M_{\mathrm{s}}^{2}-1\right)
$$

can be equated to the predictive equation for peak overpressure, Eq. (22). Here, $M_{\mathrm{s}}$ is the Mach number for the shock moving through still air. Because $\mathrm{d} t_{\mathrm{a}} / \mathrm{d} r=1 / M_{\mathrm{s}}$, the resulting expression can be integrated to obtain a closed form expression for the time of arrival, $\bar{t}_{\mathrm{a}}$. The expression for the peak overpressure, Eq. (20), when substituted into Eq. (23) and 
integrated from the muzzle to the field point, $r$, becomes

$$
\bar{t}_{\mathrm{a}}-\bar{t}_{0}=X(\bar{r})-\frac{A^{\prime}}{2} \ln \left[2 X(\bar{r})+2 \bar{r}+A^{\prime}\right],
$$

in which

$$
\begin{aligned}
& X(\bar{r})=\sqrt{\bar{r}^{2}+A^{\prime} \bar{r}+B^{\prime}}, \\
& A^{\prime}=\frac{2 \gamma A}{\gamma+1}, \quad B^{\prime}=\frac{2 \gamma B}{\gamma+1},
\end{aligned}
$$

and $\gamma=1.4$ is the specific heat ratio for ambient air. The initial time value, $\bar{t}_{0}$, takes into consideration the nature of the formation of the blast wave and may vary with the angle. The zero value for time corresponds to the projectile exiting the muzzle.

The time-of-arrival expression, Eq. (24), can be used with the determined constants $A, B, \gamma$, and the time-of-arrival data to obtain the value, $t_{0}$, by a least squares fit

$$
\begin{aligned}
\bar{t}_{\mathrm{a}}= & X(\bar{r}) \\
& -0.064 \ln [2 X(\bar{r})+2 \bar{r}+0.128]-0.17,
\end{aligned}
$$

in which

$$
X(\bar{r})=\sqrt{\bar{r}^{2}+0.128 \bar{r}+0.0074} .
$$

Least squares fits were also tried to see if a directional dependence existed for the scaled time of arrival. The directional dependence was negligible.

Figure 5 shows the fitted curve for $\bar{t}_{\mathrm{a}}$ as a function of the scaled length with the scaled time-of-arrival data. A logarithmic scale was used to emphasize comparisons for the shorter scaled distances. The agreement of the data with the fitted time of arrival is satisfactory.

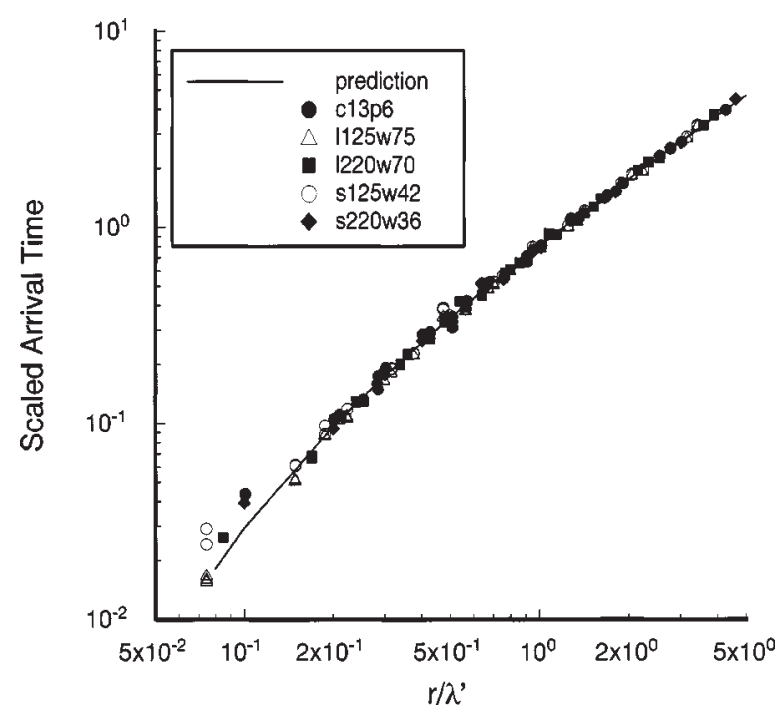

Fig. 5. Predicted $\bar{t}_{\mathrm{a}}$ compared with the observed $\bar{t}_{\mathrm{a}}$.

\subsection{Impulse data presented with different scaling approaches}

Similarly as for the peak overpressure data, the impulse data along selected direction rays $\left(30^{\circ}, 90^{\circ}\right.$, and $150^{\circ}$ ) are scaled with both the ideal point source scale length and the energy efflux scale length. Figure 6 shows the impulse data obtained along a $30^{\circ}$ ray when scaled using the constant energy efflux assumption. The data do not appear to be well correlated using energy efflux scaling. The carbine (c13p6) empties rapidly and has the lowest impulse values while the long barrel Magnum with the largest projectile (1220w70) empties its barrel more slowly and is among the data with the higher values for the impulse. The same trends occur for data taken at other angles and with approximately the same spread in the data values (Fansler [7]).

In general, constant energy efflux scaling for the impulse data yields inferior correlations compared to the corresponding correlations for the peak overpressure data. This result seems physically reasonable because the peak value is more responsive to the early part of the energy efflux history while the shape of the wave would more strongly depend upon the complete energy efflux history, which is partially described by the blowdown parameter value.

Figure 7 shows the impulse data obtained along a $30^{\circ}$ ray when point source scaled. Nearer the gun muzzle, the impulse decreases slowly at first and then descends much more rapidly for the longer distances.

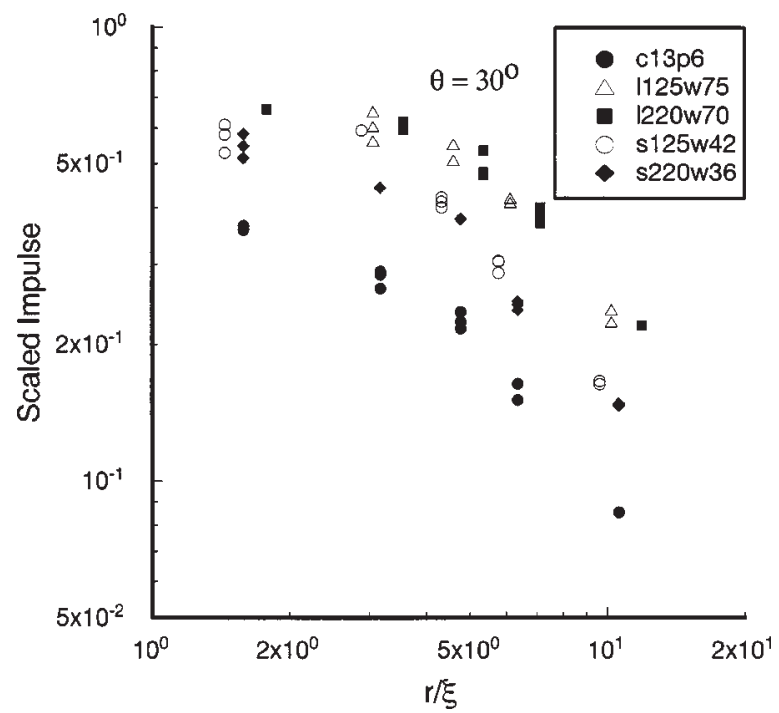

Fig. 6. Scaled impulse versus $r / \xi$ for $\theta=30^{\circ}$. 


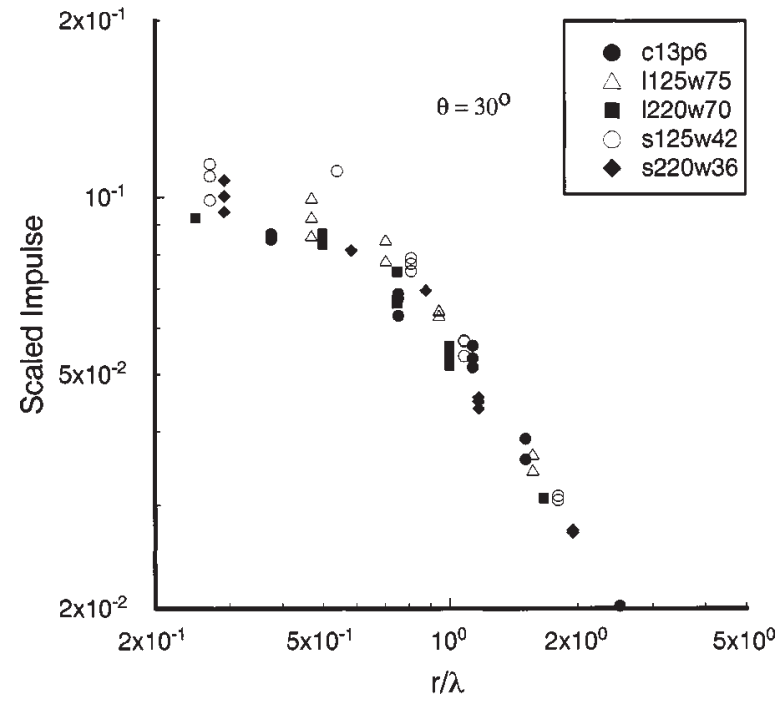

Fig. 7. Scaled impulse versus $r / \lambda$ for $\theta=30^{\circ}$.

Apparently, energy flux flowing out of the gun continues to add to the blast as the wave passes over the first gauges used in this investigation and as the energy flux decreases, the curve steepens. The data are well correlated by point source scaling. The correlation is improved for the larger distances, which would be expected because almost all of the available energy would have been deposited by the time the front of the wave reaches the larger distances, while at the smaller distances, the various guns would have deposited different fractions of their total available energy. For the larger scaled distances, the correlation for the impulse at $30^{\circ}$ is superior to the correlation obtained from the scaling investigation of the peak overpressure. The impulse is the time integral of the positive phase of the overpressure and would be expected to be a better indicator of the total energy deposited into generating the blast wave as compared to the peak overpressure. Nevertheless, the data points are, on the whole, slightly lower for the more slowly emptying guns, as occurs for the peak overpressure data.

The data values for other angles show less correlation and descend more slowly than observed for other angles (Fansler [7]). The impulse data values for point source scaling are better correlated than the impulse data values for constant energy efflux scaling. Accordingly, only the data that are point source scaled will be least squared fitted using the barrelemptying parameter to improve the fit.

\subsection{Modeling and fitting impulse data}

With the observation noted previously, candidate fitting equations may be proposed. The best candidate equation with its fitting coefficients are

$$
\bar{I}=0.0146\left(\frac{\lambda_{\mathrm{i}}^{\prime}}{r}\right)^{b_{\mathrm{i}}(\theta)},
$$

in which

$$
b_{\mathrm{i}}(\theta)=0.954-0.585 \sin \frac{\theta}{2},
$$

and

$$
\lambda_{\mathrm{i}}^{\prime}=\beta\left[1+0.444 \frac{\sin (\theta / 2)}{\delta_{\lambda}}\right] \lambda .
$$

Note that the scaling length for the gun blast impulse differs from the scaling length for the peak overpressure, with the emptying parameter characterizing the gun blast, $\delta_{\lambda}$, being applied more strongly to the rear of the gun. The least square fit was obtained using the logarithm of the impulse values as was done for the peak overpressure data. The RMS value was equal to 0.216 , which is only a little larger than the RMS found for the peak overpressure data. Other more complicated fits were tried to account for the change in the slope with distance for the data forward of the gun, but only marginal improvements in the RMS values were found. The dimensionless form of the impulse for gun blast is then

$$
\bar{I} \equiv I a_{\infty} /\left(\lambda_{\mathrm{i}}^{\prime} p_{\infty}\right) .
$$

Unlike the presentation of the peak overpressure, when scaled with the modified point source length, the impulse data scaled by its special modified point source length must be presented with the polar angle as a parameter because $b_{\mathrm{i}}(\theta)$ in Eq. (27) varies with the polar angle. Figure 8 shows the impulse data scaled with the modified point source length for impulse together with the fitted curve for the $30^{\circ}$ polar angle. The fitted curve is a compromise that, for small distances, is too high at first and then transitions to a region where it is too low and then becomes too large again.

The flow processes are too complex to assume a form for the positive phase duration and to obtain an accurate estimate of its value from some simple assumptions. Moreover, accurate values of the positivephase-duration data are more difficult to obtain because the overpressure varies slowly as the zero value of overpressure is approached, with smaller superimposed waves from turbulence in the flow resulting in large amounts of uncertainty for the time for the zero value of overpressure in the wave. 


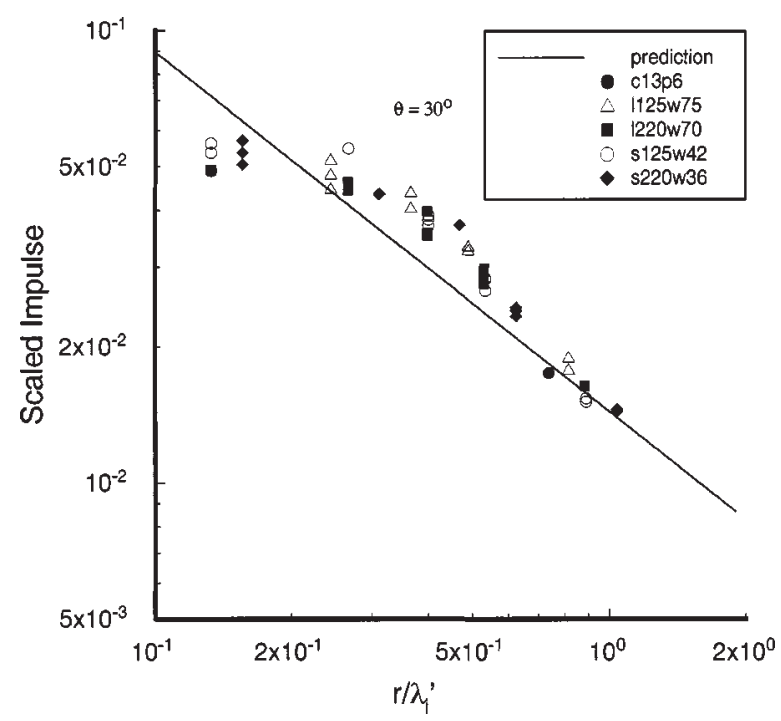

Fig. 8. Scaled impulse versus $r / \lambda_{\mathrm{i}}^{\prime}$ for $\theta=30^{\circ}$ with least squares fit.

\section{Conclusions and recommendations}

Investigators have advocated particular approaches for describing gun muzzle blast. This investigation examines two principal approaches that use scaling ideal blast waves as a basic starting point. The data available consist of detailed overpressure records for weapons with a wide variety of locations, projectile weights, propellant weights, muzzle velocities, and barrel lengths. From the data, the peak overpressure, the time of arrival, and the impulse obtained by integrating the positive value of the overpressure wave with time are obtained. The peak overpressure data are scaled using both ideal scaling models: the instantaneous energy release model and the constant energy deposition model. Neither of the scaling approaches gives a clearly superior correlation of the data.

Two parameters that characterize gun blast are introduced for modification of the basic scaling approaches to improve data correlation. One is called the blow-down or emptying time parameter, while the other parameter gives the position of the Mach disc in terms of calibers. For the peak overpressure data scaled with the energy efflux length, the Mach disc position parameter noticeably improves correlation of the data, while the blow-down parameter value improves the correlation negligibly. For the data scaled with the point blast scaling length, the blow-down parameter is important for improving correlation, and the effect of the Mach disc location may be neglected. Slower emptying times result in an inefficient con- version of energy into the muzzle blast wave that reduces the peak overpressure. Both modified scaling approaches give similar improvements in correlation of the peak overpressure data. The modified point mass model was selected because the basic point mass model yielded a superior correlation for the impulse data. A two-term equation for predicting peak overpressure was selected that permits the development of a closed form expression describing the time of arrival and also approximates the nonlinear wave behavior near the muzzle.

The impulse data are also scaled with the two ideal models. The instantaneous energy release model correlates the data well to the front of the gun with less correlation as the polar angle is increased. The impulse decreases more slowly with distance for increasing polar angle. The constant energy deposition model does not correlate the impulse data as well as the point source model, particularly to the front of the gun. The point source model modified by the use of the gun-emptying parameter is used to develop a least squares fit function describing the data. The length scale developed for impulse data differs in form from the length scale for peak overpressure and depends upon the polar angle, $\theta$. The RMS error obtained with the impulse data is only slightly larger than the error obtained for the peak overpressure data.

A prediction method for the positive phase duration is left for further investigations. More needs to also be known about the detailed flow processes in gun blast. Computational fluid dynamics techniques could be used to investigate the flow of energy from the front to the rear part of the wave and yield more insight into gun blast phenomena.

\section{Acknowledgements}

I wish to thank William Thompson, John Carnahan, and Brendan Patton (all who then worked at ARL) for their assistance in conducting many of these experiments and obtaining the data.

\section{References}

[1] B.F. Armendt and J. Sperrazza, Air blast measurements around moving explosive charges, Part III, BRL-MR 1019, U.S. Army Ballistic Laboratory, Aberdeen Proving Ground, MD, 1956.

[2] W.E. Baker, Explosions in Air, University of Texas Press, Austin, TX, 1973, pp. 54-77. 
[3] H.L. Brode, Numerical solutions of spherical blast waves, J. Appl. Phys. 26 (1955), 766-775.

[4] J. Corner, Theory of the Interior Ballistics of Guns, Wiley, New York, NY, 1950, pp. 339-383.

[5] J.I. Erdos and P. DelGuidice, Gas dynamics of muzzle blast, AIAA Journal 13 (1975), 1048-1055.

[6] K.S. Fansler, Dependence of free field impulse on the decay time of energy efflux for a jet flow, Shock and Vibration Bulletin, Bulletin 56, Part 1, The Shock and Vibration Center, Naval Research Laboratory, Washington, DC, 1986, pp. $203-$ 212.

[7] K.S. Fansler, Description of muzzle blast by modified ideal scaling models, ARL-TR 1434, U.S. Army Ballistic Research Laboratory, Aberdeen Proving Ground, MD 21005, 1997.

[8] K.S. Fansler and E.M. Schmidt, The prediction of gun muzzle blast properties utilizing scaling, ARL-TR 227, U.S. Army Ballistic Research Laboratory, Aberdeen Proving Ground, MD 21005, 1983.

[9] K.S. Fansler, W.P. Thompson, J.S. Carnahan and B.J. Patton, A parametric investigation of muzzle blast, ARL-TR 227, U.S. Army Ballistic Research Laboratory, Aberdeen Proving Ground, MD 21005, 1993.

[10] C.W. Heaps, K.S. Fansler and E.M. Schmidt, Computer implementation of a muzzle blast prediction technique, Shock and Vibration Bulletin, Bulletin 56, Part 1, The Shock and Vibration Center, Naval Research Laboratory, 1986, pp. 213 230.
[11] B. Hopkinson, British Ordnance Minutes, No. 13565, 1915.

[12] J. Kietzman, K.S. Fansler and W.G. Thompson, Muzzle blast from 105 mm M735 round, ARLBR-MR 3957, U.S. Army Ballistic Research Laboratory, Aberdeen Proving Ground, MD 21005, 1992.

[13] L.L. Pater, Gun blast far field peak overpressure contours, TR 79-442, Naval Surface Weapons Center, Dahlgren, VA, 1981.

[14] B.T. Reynolds, Muzzle blast pressure measurements, Report No. PMR-21, Princeton University, 1944.

[15] E.M. Schmidt, E.J. Gion and K.S. Fansler, Analysis of weapon parameters controlling the muzzle blast overpressure field, in: 5th Int. Symp. on Ballistics, Toulouse, France, 1980.

[16] F. Smith, A theoretical model of the blast from stationary and moving guns, in: First Int. Symp. on Ballistics, Orlando, FL, 1974.

[17] G. Soo Hoo and G.R. Moore, Scaling of naval gun blast peak overpressures, TN-T7-72, Naval Surface Weapons Center, Dahlgren, VA, 1972.

[18] P. Westine, The blast field about the muzzle of guns, Shock and Vibration Bulletin 39 (1969), 139-149.

Received 6 May 1994; Revised 5 November 1997 

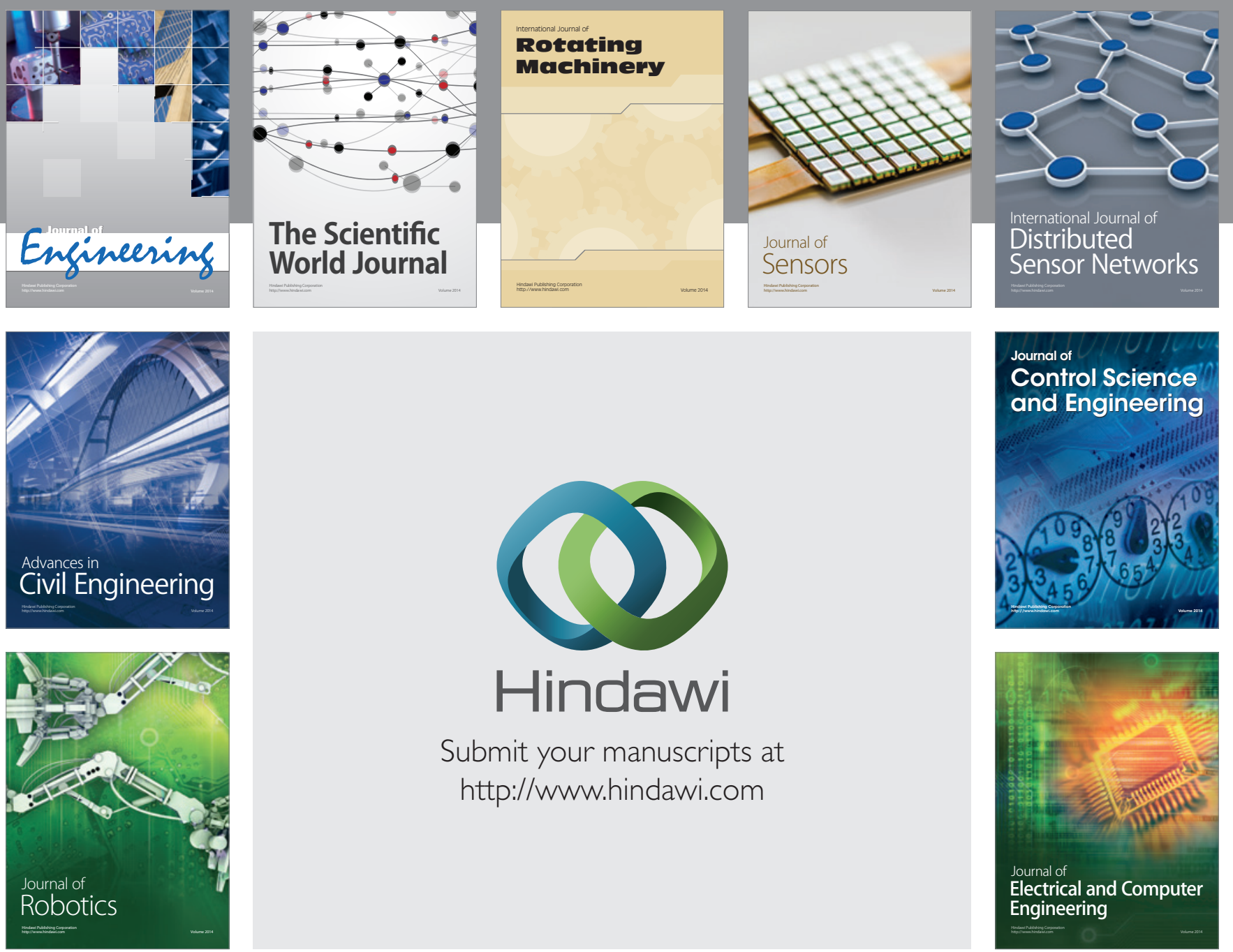

Submit your manuscripts at

http://www.hindawi.com
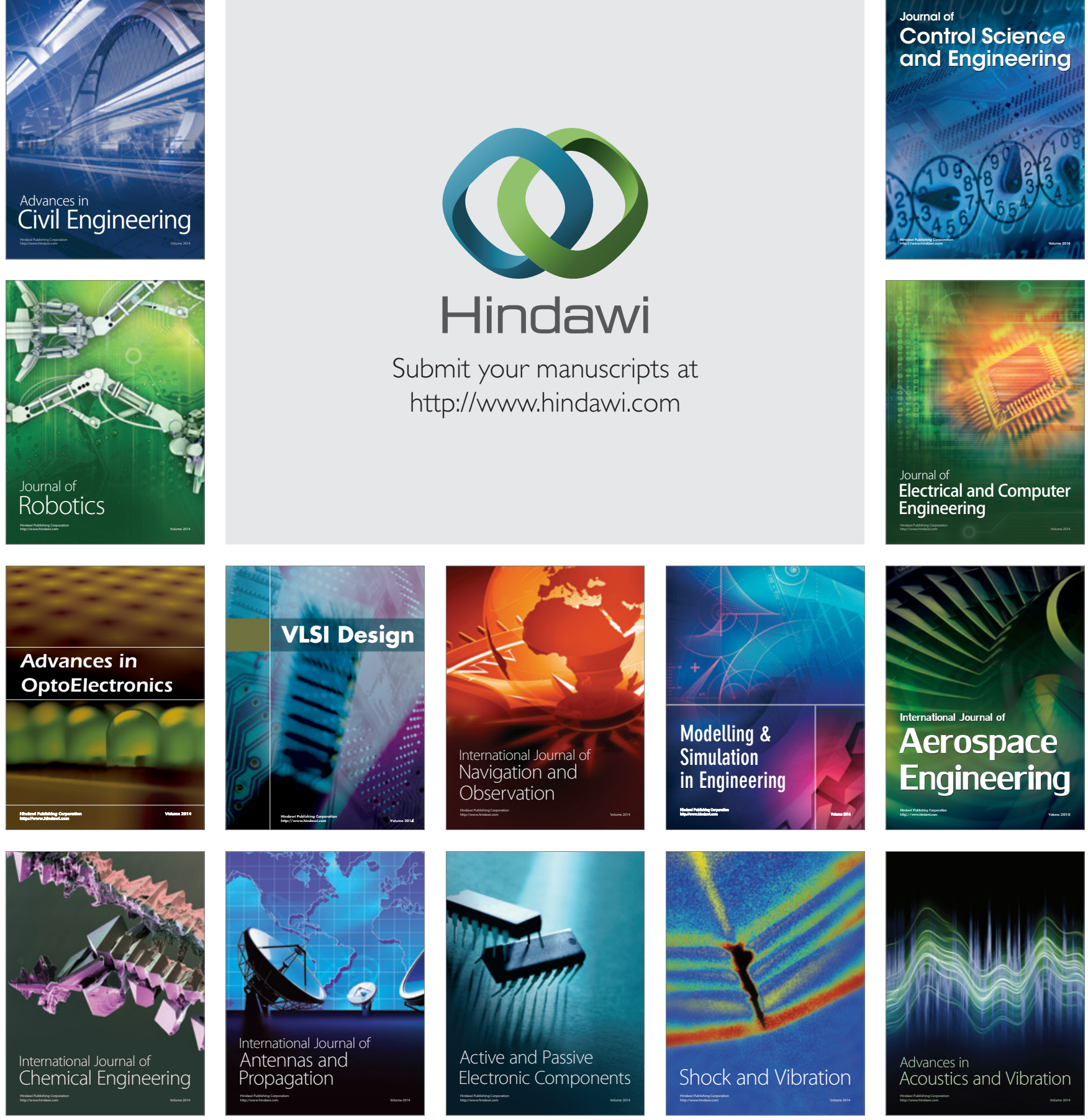